\title{
Entropy image analysis of primary atomization for a coaxial air-water jet submitted to acoustics at variable ambient pressure
}

\author{
HERRERA Rafael ${ }^{1}$, BAILLOT Francoise ${ }^{1}$, BLAISOT Jean-Bernard ${ }^{* 1}$ \\ ${ }^{1}$ CORIA, CNRS, Normandie Université, UNIROUEN, INSA ROUEN, Saint-Étienne-du-Rouvray, \\ France \\ *Corresponding author email: blaisot@coria.fr
}

\begin{abstract}
Statistical entropy image analysis is used here to quantify the primary atomization area of a coaxial water-air jet submitted to a high amplitude acoustic standing field at ambient pressure ranging between 1 and $5 \mathrm{~atm}$. The statistical entropy, i.e. Shannon entropy, is computed from the probability of three states in spray images, i.e. liquid jet, dispersed liquid phase and surrounding gas. One region of interest, called the primary atomization region, is defined where the three states have non null probability. The geometric characterization of this region helps in comparing the effect of acoustics on the atomizing jet for varying parameters such as ambient gas pressure or acoustic pressure level.
\end{abstract}

\section{Keywords}

Probability, entropy, atomization, acoustic radiation pressure

\section{Introduction}

Thermoacoustic instabilities in liquid rocket engines represent a big challenge to overcome in new rocket design. Indeed, pressure fluctuations created by the combustion process can be coupled with combustion chamber acoustic modes leading to a high perturbation of combustion heat release. Thermoacoustic instability can produce a high damage that can eventually lead to the destruction of the rocket engine [1]. One of the mechanism implied in this coupling is the interaction between acoustics and atomization that is considered in the present work.

Our research objectives aims at increase our understanding of non-linear effects of linear acoustic on the atomization process. Previous works demonstrated that the atomization of air assisted liquid jets can be drastically modified when submitted to a high frequency $(\simeq 1 \mathrm{kHz})$ and high amplitude $(\leq 12 \mathrm{kPa})$ controlled acoustic field ([2], [3]). The actual approach covers a large range of two phase flows regimes, going from low Weber number (Rayleigh regime) up to high Weber number (fiber regime) adding the effect of the ambient gas pressure.

To perform this experimental study, an original acoustic cavity (MARACA: Moderate Ambient pRessure Acoustic Chamber) was developed at CORIA lab. This cavity was characterized numerically and experimentally. Characterization of cylindrical liquid jets flattening and deviation as well as of single droplets deviation was studied by [2] and [4]. They implemented a description of the modification of the whole spray assembly by the acoustics by means of statistical methods showing droplets clustering in the acoustic field. Some of those phenomena were in parallel quantified using theoretical model of non-linear quantities obtained from linear acoustic (Radiation pressure and radiation force) for objects with circular geometry (Circular cylinder and sphere) based on the model found in [5] and [6]. Thus, deviation angle for non-deformed objects and acoustic level threshold for the flattening of liquid cylindrical jet was determined. From the acoustic model it was observed a modification of the acoustic effects as a function of the ambient gas density.

The present works aim to analyse the influence of the gas density on a coaxial jet water/air atomization submitted to acoustics. Atomization perturbations are here quantified by means of visualization techniques. A statistical description based on probability images and entropy analysis is used to analyse the modifications on the atomizing jet. 


\section{Experimental bench}

This section presents the elements of the experimental bench used for this study (see figure 1). The experimental bench MARACA is composed of 4 main parts: the acoustic resonant cavity, the liquid-gas system, the acoustic acquisition system and the optical system successively described below.

\section{Resonant acoustic cavity}

The acoustic resonant cavity is a closed stainless steel parallelepipedic cavity presenting four acoustic accesses connected to loudspeakers, four lateral optical accesses (quartz windows) and an injection system at the top. Each loudspeaker Beyma CP-850ND is enclosed in a pressurized cylindrical cavity to maintain the static pressure balance on both sides of its membrane. A schematic view of the closed resonant cavity is shown in Fig. 1(a).

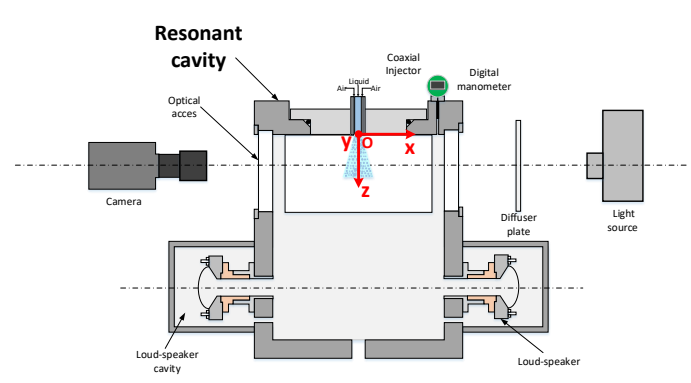

(a)

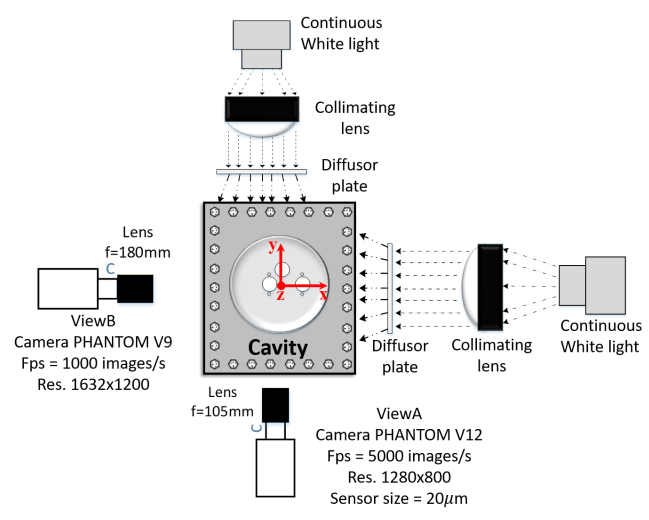

(b)

Figure 1. (a) Schematic view of the resonant cavity and (b) view A and view B optical configurations.

The dimensions of the acoustic cavity $(\mathrm{WxHxL})$ are chosen in order to favour 2T acoustic mode in the cavity along $x$ axis. Axes $x, y$ and $z$ are defined in Fig 1(a). The origin $O$ of the Cartesian coordinate system $(O, \vec{x}, \vec{y}, \vec{z})$ is centred on the inner top of the acoustic chamber.

Table 1. Injection conditions

\begin{tabular}{|c|c|c|}
\hline \multicolumn{3}{|c|}{$P=1,3$ and 5 $\mathrm{atm}$} \\
\hline$R e_{l}$ & $W e_{g}$ & name \\
\hline 2500 & 9 & Reynolds axisymetric \\
3000 & 40 & Reynolds non axisymetric \\
2900 & 60 & shear breakup \\
3200 & 129 & membrane \\
3200 & 224 & fiber \\
\hline
\end{tabular}

\section{Liquid-gas injection and operating conditions}

The injection system is composed of a liquid (water) line and a gas (air) line, feeding a coaxial liquid-gas injector of inner liquid diameter $D_{l}$ and outer gas diameter $D_{g}$.

A home-made LabView program is used to control the fluid flows. The injection cavity top comprises 3 injection ports and 6 acoustic measurement points located near the injection ports.

Operating conditions are given in table 1. Three values were considered for the ambient pressure in the acoustic cavity, i.e. $P=1 \mathrm{~atm}, 3 \mathrm{~atm}$ and $5 \mathrm{~atm}$. Liquid and gas flow rates were adjusted to maintain the liquid Reynolds number $R e_{l}=\frac{\rho_{l} u_{l} D_{l}}{\mu_{l}}$ and the gaseous Weber number $W e_{g}=\frac{\rho_{g} u_{g}^{2}}{\sigma / D_{l}}$. 


\section{Acoustic field}

Sinusoidal signals are digitally generated at a fixed frequency $\simeq 1 \mathrm{kHz}$ and variable amplitude. The sampling frequency for signal generation is fixed to $70 \mathrm{kHz}$. The signal envelope is composed of three main parts: two linear ramps at start and end, and a plateau in the middle, each with a duration of $\simeq$ $300 \mathrm{~ms}$. The amplitude at the plateau is varied to deliver the wanted acoustic level.

The acoustic measurement is done by means of dynamic pressure piezoelectric transducers PCB106b connected to a ICP conditioner. The sample frequency chosen for the acoustic acquisition is $52 \mathrm{kHz}$. Acoustic simulations by means of COMSOL multiphysics has been done to determined different eigenmodes for the geometry used for the experiments. In these simulations, walls are considered as completely reflecting and a harmonic acceleration is imposed for the loudspeaker membrane. Results show, for the frequency $\approx 1 \mathrm{kHz}$, a planar standing wave in the $x$ direction corresponding to the 2nd transverse (2T) mode of the cavity. Three locations are of interest in the acoustic field, i.e. the pressure antinode (PAN), the intensity antinode (IAN) and the velocity antinode(VAN). PAN, IAN and VAN can respectively be found at $x=0, \frac{x}{W}=\frac{1}{8}$ and $\frac{x}{W}=\frac{1}{4}$ (see Fig. 2(a)). All results in this paper are for an injection placed at IAN.

The acoustic field in the cavity is characterized experimentally by measurements made with pressure sensors flush-mounted in the cavity top. Acoustic signal is analyzed by the power spectrum density (PSD). Then, a band-pass elliptic filter is used to extract the fundamental and harmonic components from the raw signal. Raw signal, fundamental and second harmonic are reported in Fig. 2 and compared with simulations, the fundamental mode being the most energetic one. Filtered experimental signals agree well with simulations showing the good performance of MARACA bench to produce the $2 \mathrm{~T}$ mode.

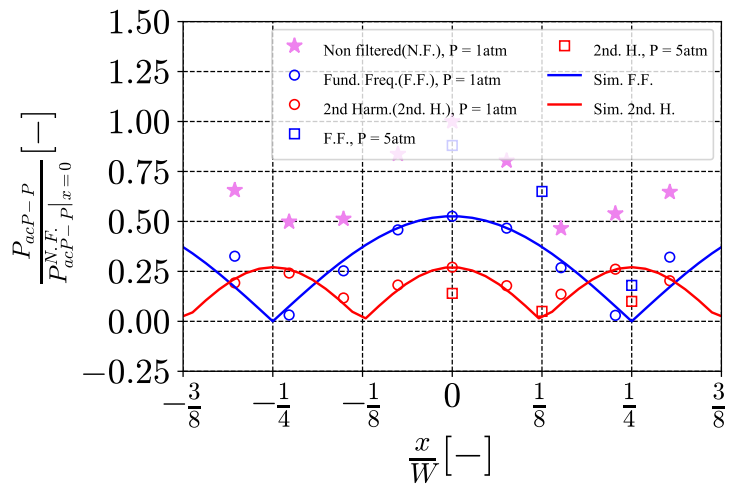

(a)

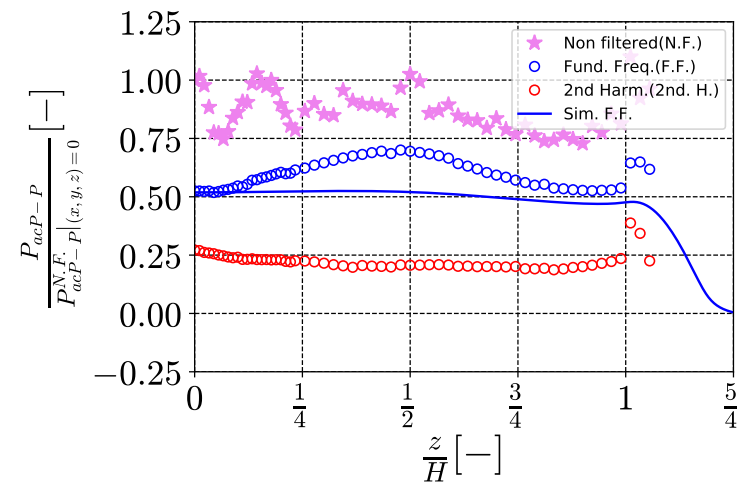

(b)

Figure 2. Spatial acoustic pressure characterization along acoustic axis : (a) along $O x$ and (b) along $O z$.

In the following, acoustic effects are presented as a function of the radiation pressure amplitude term, which take into account the kinetic energy of the acoustic wave: $P_{r a d}=P_{a c}^{2} /\left(\rho_{g} c_{0}^{2}\right)$ where $P_{a c}$ is the acoustic amplitude and $c_{0}$ is the speed of sound.

\section{Image acquisition}

Diffuse backlight visualization was performed to characterize the atomization process. The optical setup consists of a white light source that is used as a background, a camera and an objective to capture images of the jet. A diffuser plate is placed between the light source and the object (the jet) in order to ensure an homogeneous grey-level for the background. A schema of this configuration is shown in Fig. 1(b). In such a backlight configuration, objects appear as black over a white background. Three camera setups were used in the experiments: A - MatrixVision BlueCOUGAR CCD camera (2448x2050 pixels, $10 \mathrm{fps})$, magnification $\gamma=0.086$, stroboscope light source $(\delta t \simeq 10 \mu s)$. B - Phantom V9 high-speed camera (1632x1200 pixels, 1000 fps), C - Phantom V12 high-speed camera (1280x800 pixels, $5000 \mathrm{fps})$,

A first analysis was done without acoustics to quantify the effect of the ambient pressure on the 
atomization process. Even though the images of the sprays are qualitatively similar whatever the ambient pressure when Reynolds and Weber number are the same, it was necessary to provide a quantitative analysis to assess objectively the likeliness of these processes. This was done by a statistical analysis performed on non-correlated image series recorded with camera setup $\mathbf{A}$ in view A configuration (cf. Fig. 1(b)).

To estimate the effect of acoustics on the atomizing jet, time-resolved image series were recorded and time-resolved as well as statistical analyses were done on these images. The two camera setups $\mathbf{B}$ and $\mathbf{C}$ were used, respectively on view $A$ and view $B$ configurations, in order to get simultaneous visualization of the spray in two perpendicular directions.

\section{Image processing}

The objective of image processing is to localize the liquid-gas interface in order to separate liquid and gas phases on the images. Raw images are first pre-processed through a normalization procedure to compensate background inhomogeneities. Normalized imaged are segmented and continuous (jet) and dispersed (droplets) liquid parts are dissociated.

\section{Normalisation}

Diffuse backlight illumination images are characterized by the transmission of light through the objects. Image normalization is a way to compute the transmission function characterizing these objects, thus removing possible defects of the background. A reference background image $I_{\text {back }}$ and a reference noise image $I_{N}$ are used to compute $I_{\text {norm }}$ (see Eq. 1). Background image $I_{b a c k}$ is obtained with no object in the field of view and noise image $I_{N}$ is obtained with no light. An additional parameter $\alpha$ is used to compensate from shot-to-shot fluctuations of the intensity of the illumination source and $\bar{I}_{\text {back }}$ is the mean level of the background image.

$$
I_{\text {norm }}=\alpha \bar{I}_{\text {back }} \frac{I_{\text {raw }}-I_{N}}{I_{\text {back }}-I_{N}}
$$

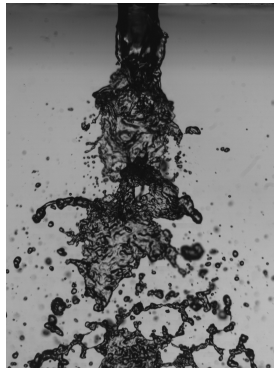

(a)

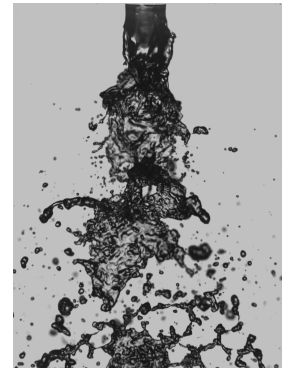

(b)

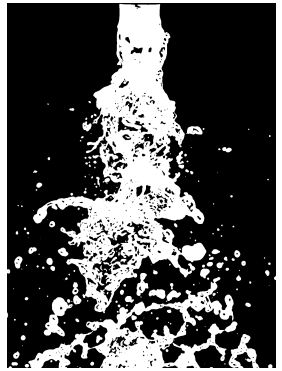

(c)

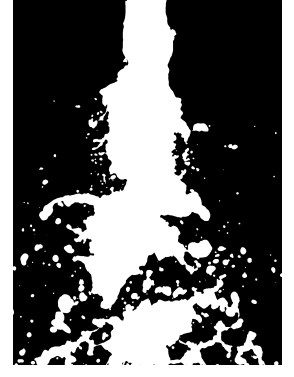

(d)

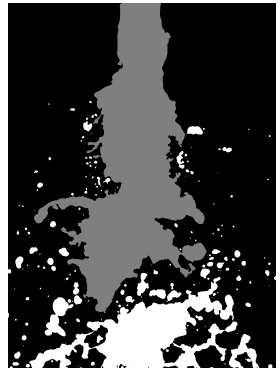

(e)

Figure 3. image processing: (a) raw image; (b) normalized image; (c) binary image; (d) filled-hole image; (e) labelled image. View $\mathrm{B}$ for injection for $R e_{l}=3200$ and $W e_{g}=129$.

\section{Image segmentation}

The normalized images present an almost uniform background, allowing image segmentation to be performed by a simple global threshold technique. An accurate gray level threshold to perform this operation is obtained by the Otsu method. This method is based in the identification of two class in the gray level histogram. The two-level image obtained from thresholding often presents liquid parts with holes. These holes are induced by the refracted light through the liquid that generates bright spots in the image (see images (b) and (c) in Fig. 3). To overcome this problem, a morphological operation was used to fill these false holes. 


\section{Image labelling}

Image labelling consist in a identification of the connected sets of pixels in a binary image. Such a set, usually called an object, is a set of pixels within a given contour. Labelling is performed by a morphological operation that assign to each object in the binary image a different label, here a grey level. It is used to identify the liquid attached to the nozzle orifice, called the jet or the liquid core and the dispersed liquid phase. This is shown in Fig. 3(e) where the grey object corresponds to the liquid jet and white objects correspond to the dispersed liquid phase.

The three states of interest are the jet, the dispersed liquid phase and the background (gas phase). The probability for each state is determined by counting the frequency of occurrence of each state for a given pixel $(i, j)$, giving the local probability $P_{s}(i, j)$ of this state. An example of jet probability image and dispersed-phase probability image is given in Fig. 4(a) and 4(b) respectively in grey level where black level correspond to $P_{s}=0$ and white to $P_{s}=1$. Indeed, the probability of one state can be deduced from the value for the other two states.

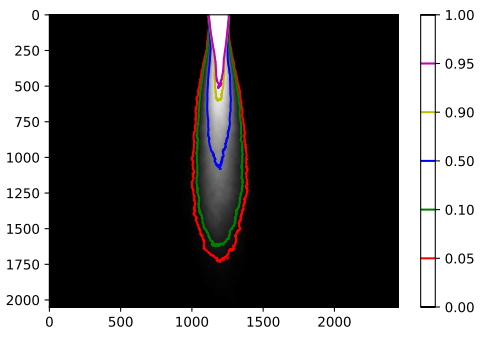

(a)

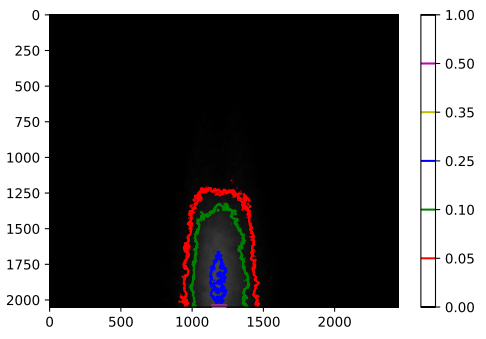

(b)

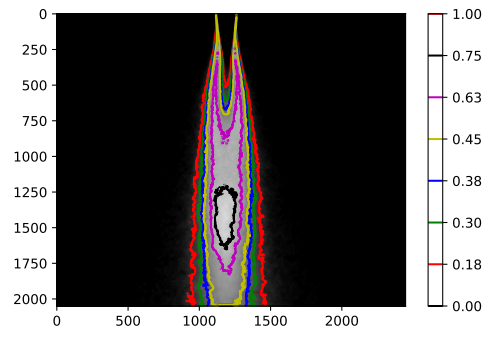

(c)

Figure 4. Examples of the probability ( 0 is black and 1 is white) and entropy: (a) the jet probability of presence; (b) the dispersed phase probability of presence; (c) the entropy map. Injection for $R e_{l}=3200$ and $W e_{g}=129$.

\section{Entropy map}

Entropy based images has been used previously by [7] to determine the characteristics length scales of the jet interface in the case of a diesel injection and [8] in the case of a cavitating liquid jet. They proved that entropy based image is an accurate method to describe statistically the primary atomization zone. This statistical entropy, i.e. Shannon entropy, is computed from the probability of the three states introduced in the previous section. It is expressed as :

$$
E=\frac{-\sum_{s=1}^{s=n} P_{s} \ln P_{s}}{\ln n}
$$

where $P_{s}$ is the probability of a given state $s$ and $n=3$ is the number of states which are: the jet, the dispersed phase and the air (background). An entropy map is shown in Fig. 4(c). Low entropy level regions (in black or dark grey) correspond to a very stable state in this region over the image series. At the opposite, high entropy levels correspond to a highly variable state in the region. One region of interest is the one for a normalized entropy greater than 0.63 , which is a region where the three states (jet, dispersed phase and gas phase) can be found. This region is called the primary atomization region as it is located where the fragmentation of the jet occurs.

The breakup length and droplet size distribution are measured from the image series. Combined with the jet probability image, the dispersed phase probability image and the entropy image, these data allow the two phase flow regimes to be quantified. In this paper the focus is put on statistical properties of the liquid jet, of the dispersed phase and of the region of primary atomization in terms of length, width and area.

\section{Image regions analysis}

The expansion of the jet is characterized from the jet probability image through the criterion $P_{\text {jet }} \geq$ $10 \%$, giving the region $R_{j}$ where the jet is present more than $10 \%$ of the time. The same is done for the dispersed phase (i.e. $P_{d} \geq 10 \%$ giving the region $R_{d}$ ) and for the primary atomization area 
with the criterion $E \geq 0.63$, giving the region $R_{a}$. The local width of these areas is computed as functions of $z$, giving the jet width profile $l_{j}(z)$, the dispersed-phase width profile $l_{d}(z)$ and the primary atomization width profile $l_{a}(z)$. Examples of such profiles are shown in Fig. 5. The jet width value at nozzle orifice location $(z=0)$ is obviously equal to $D_{l}$ and increase up to $\simeq 2-2.5 D_{l}$ at downstream location $z \simeq 4-8 D_{l}$. The width of atomization area start value is 0 and increase up to $2 D_{l}$ at $z \simeq 5-7 D_{l}$. Whereas ambient pressure level does not modify significantly $l_{j|d| a}(z)$ curves, adding acoustics results in a modification of these curves that start increasing closer to the nozzle orifice.

The mean lengths $L_{j|d| a}$ and the areas $A_{j|d| a}$ of these regions are deduced from $l_{j|a| d}(z)$. These areas are normalized by the liquid jet exit diameter and the corresponding length, i.e. $\widetilde{A}_{j}=\frac{A_{j}}{L_{j} D_{l}}$, $\widetilde{A}_{d}=\frac{A_{d}}{L_{d} D_{l}}$ and $\widetilde{A}_{a}=\frac{A_{a}}{L_{a} D_{l}}$. These values give the mean width of these regions as a multiple of the liquid jet exit diameter $D_{l}$.

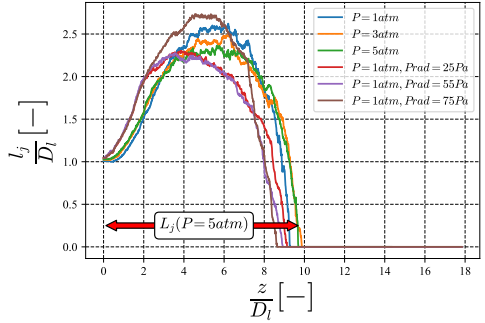

(a)

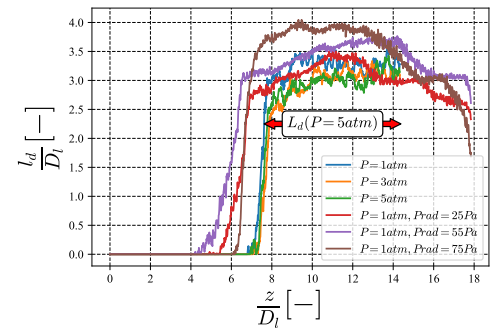

(b)

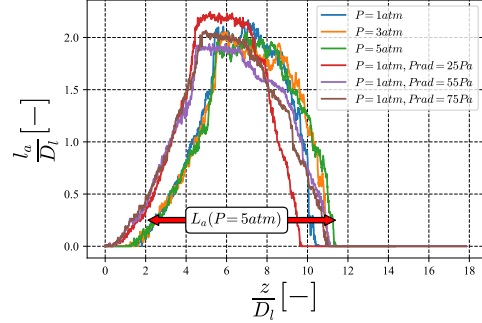

(c)

Figure 5. Vertical profiles for region width: (a) jet $l_{j}(z)$; (b) dispersed phase $l_{d}(z)$; (c) primary atomization $l_{a}(z)$. Injection at IAN for $\operatorname{Re}_{l}=3200$ and $W e_{g}=224$.

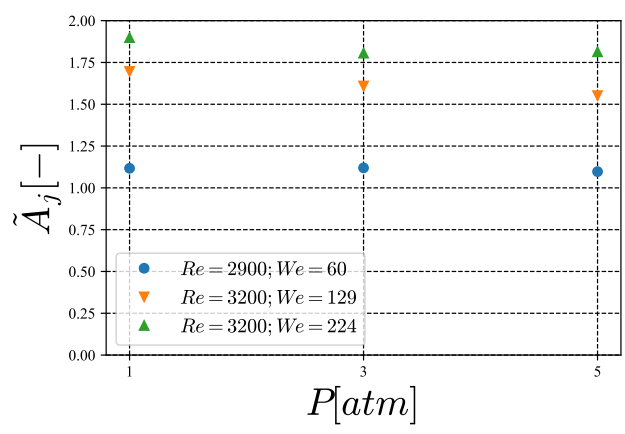

(a)

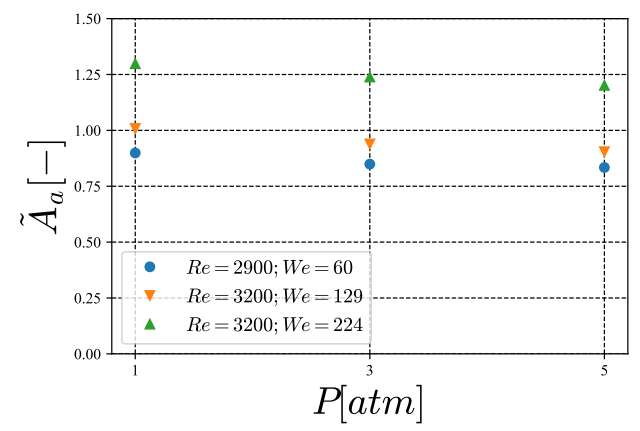

(b)

Figure 6. (a) Liquid jet expansion area and (b) primary atomization area of a coaxial jet for different two-phase flows regimes as a function of the injection pressures.

\section{Ambient pressure influence on a coaxial jet without acoustics}

Results for $\widetilde{A_{j}}$ and $\widetilde{A}_{a}$ are shown in Fig. 6 . It can be seen on this figure that as qualitatively observed, the variation of the ambient pressure $P$ does nearly not affect the jet expansion or the primary atomization area, confirming that the atomization is mainly controlled by $R e_{l}$ and $W e_{g}$ values. It is concluded that for pressures ranging in [1 $\mathrm{atm}-5 \mathrm{~atm}]$ the two phase flows regimes remains the same provided that $R e_{l}$ and $W e_{g}$ are maintained. Indeed, $\widetilde{A}_{j}$ and $\widetilde{A_{a}}$ increases monotonously with $W e_{g}$. The increase of $\widetilde{A}_{j}$ is limited for the highest Weber number, likely due to the peeling of the jet with the improvement of atomization at such a high gas Weber number. 


\section{Ambient pressure influence on a coaxial jet subjected to acoustics}

Liquid jet expansion normalized area $\widetilde{A}_{j}$ is shown in Fig. $7(\mathrm{a})$ as a function of the radiation pressure for three ambient pressures and for $W e_{g}=224$ and $R e_{l}=3200$. The larger values are obtained for $P=1 \mathrm{~atm}$. With no acoustics and for $P=1 \mathrm{~atm}$, the mean jet width is about two times $D_{l}$ and it slightly decreases with the radiation pressure. The increase of the ambient pressure accentuates the decrease of $\widetilde{A_{j}}$ for the lowest radiation pressure values, then a plateau about 1.3 is reached for $P_{\text {rad }}>50 P a$. The variation of the mean normalized liquid jet length $\widetilde{L}_{j}=L_{j} / H_{v}$ is also represented in Fig. 7(a). It remains almost equal to 0.5 for $P=1 \mathrm{~atm}$ or without acoustics whatever the ambient pressure but increases with $P_{r a d}$ as soon as the ambient pressure is greater than $1 \mathrm{~atm}$. Indeed, the acoustics reduces the fragmentation of the jet, resulting in the lengthening of the liquid core. This can be related to a competition between aerodynamic and acoustic radiation forces, the latter acting as a cohesion force on the liquid jet as surface tension does for such high Weber values.

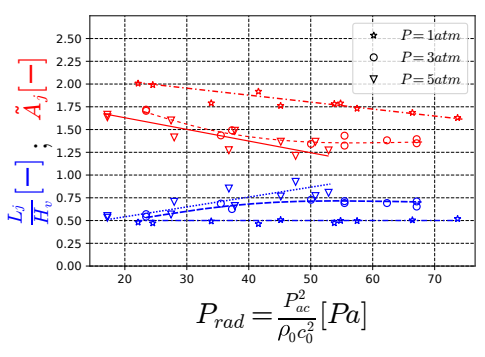

(a)

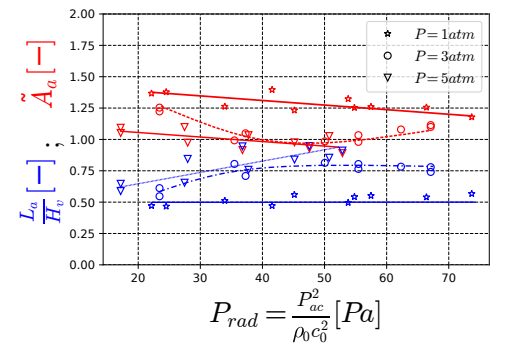

(b)

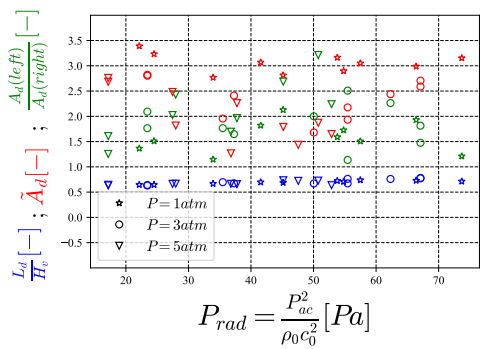

(c)

Figure 7. Liquid jet (a), primary atomization (b) and dispersed phase (c) region length and area. View $A$ for injection at IAN for $W e_{g}=224$ and $R e_{l}=3200$.

The normalized primary atomization area and atomization length are shown in Fig. 7(b). Similar trends as for $\widetilde{A}_{j}$ are observed for $\widetilde{A}_{a}$, i.e. the larger value for $P=1 \mathrm{~atm}$ and without acoustics, a slight decrease at $P=1 \mathrm{~atm}$ and a more pronounced decrease for a higher ambient pressure. Likewise, $L_{a}$ remains equals to $0.5 H_{v}$ for $P=1 \mathrm{~atm}$ and increases with $P_{\text {rad }}$ up to $P_{\text {rad }}=50 P a$. Obviously, by definition $L_{a}$ and $L_{j}$ extent are similar as the primary atomization area is linked to the presence of the jet and atomization almost starts at $z=0$ as can be seen in Fig. 5(c). The lower value of $\widetilde{A_{a}}$ for $P>1 \mathrm{~atm}$ and its decrease with $P_{\text {rad }}$ indicates a faster transition from continuous to dispersed phases. The normalized area of the dispersed phase $\widetilde{A_{d}}$ (Fig. $7(\mathrm{c})$ ) shows also a value not varying with $P_{\text {rad }}$ for $P=1 \mathrm{~atm}$ and a first decrease that is all the more important that ambient pressure is high. For $P_{\mathrm{rad}}>50 \mathrm{~Pa}, \widetilde{A}_{d}$ seems to stop decreasing but the discrepancies in the results for $\widetilde{A}_{d}$ prevent from establishing a clear trend in this case. The spray deviation to the nearest acoustic velocity antinode is also observed from the analysis of the dissymmetry of the dispersed phase distribution. Indeed, the ratio between dispersed phase areas at the left and right hand side of the jet axis (green symbols in Fig. 7(c)) shows a clear clustering of droplet on the left hand side of the jet as $P_{\text {rad }}$ increases. Here again, this is accentuated by an increase of the ambient pressure and this effect is observed up to $P_{\text {rad }} \simeq 50 P a$.

These results shows that acoustics have an effect on the atomization process. However, theoretical analyses developed for a single object (a cylinder or a sphere) predict a quasi linear dependence of the effects on the radiation pressure amplitude term $P_{\text {rad }}=P_{a c}^{2} /\left(\rho_{g} c_{0}^{2}\right)$. It is evident that the relationship is more complex in the case of a variety of object of complex shapes as encountered in air-assist atomization. Also, the influence of the ambient pressure on the acoustic effects is clear and suggests that these effects are enhanced by an increase of the ambient gas density.

A kind of saturation of non linear acoustic effects seems to occur for $P_{\text {rad }}>50 \mathrm{~Pa}$. A first explanation for this can be found in the acoustic response of the chamber at high ambient pressure. Indeed, for $P>1 \mathrm{~atm}$ the contribution of the harmonic components in the acoustic pressure field is reduced, indicating a lessening of the non linear acoustic effects. This can be noticed in Fig. 2 where we remark that the amplitude of second harmonic at $P=5 \mathrm{~atm}$ is half its value at $1 \mathrm{~atm}$ in the centre of the cavity. One possible reason can be found in the motion of the loudspeaker membranes that can 
be harder when surrounding gas density increases (here by a factor up to 5) but this change in the non-linear content of the acoustic field does need further exploration.

\section{Conclusions}

A statistical approach is reported to quantify the effect of acoustics on the atomization of a air-assist injection spray under variable ambient pressure. The ambient pressure for pressures ranging in [1 $1 \mathrm{~atm}-5 \mathrm{~atm}]$ does nearly not affect the jet atomization area, provided that $R e_{l}$ and $W e_{g}$ are maintained. But increasing the ambient pressure promotes the acoustic effect. Almost all quantities related to the atomization computed from the statistical analysis show variations with the acoustic level that are all the more important that ambient pressure is high. For example the primary atomization region area slightly decreases when increasing the radiation pressure level at $P=1 \mathrm{~atm}$, and presents a faster decrease as soon as $P>1 \mathrm{~atm}$. It is also observed that acoustic non linear effects seems to saturate as ambient pressure increases. This is guessed to be linked to a harder motion of the loudspeaker membranes for higher surrounding gas density.

\section{Acknowledgements}

The authors would like to acknowledge the National Centre for Space Studies (CNES) and Normandy Regional Council for their funding supports.

\section{References}

[1] Kaess, R. and Koeglmeier, S. and Sattelmayer, T. and Schulze, M. and Oschwald, M. and Hardi, J., 2016,"HF Combustion Stability-Research Activities in Germany", SP2016-3124816, Space Propulsion Conf.

[2] Baillot, F. and Blaisot, J-B. and Boisdron, G. and Dumouchel, C., 2009,"Behaviour of an airassisted jet submitted to a transverse high-frequency acoustic field", Journal of Fluid Mechanics, 26, pp. 305-342.

[3] Ficuciello, A., 2017,"Analyse des effets acoustiques à haute fréquence/haute intensité sur l'injection coaxiale: application aux moteurs-fusées", PhD, Université de Rouen-Normandie.

[4] Ficuciello, A. and Blaisot, J-B. and Richard, C and Baillot, F., 2017,"Investigation of air-assisted sprays submitted to high frequency transverse acoustic fields: Droplet clustering", Physics of Fluids,6, pp. 67-103.

[5] King, L. V., 1934,"On the acoustic radiation pressure on spheres", Proceedings of the Royal Society of London A: Mathematical, Physical and Engineering Sciences, 147, pp. 212-240.

[6] Zhuk, A. P., 1986,"Radiation force acting on a cylindrical particle in a sound field", International Applied Mechanics, 7, pp. 689-693.

[7] Blaisot, J-B. and Yon, J., 2004,"Entropy based image analysis for the near field of direct injection Diesel jet", ILASS-Europe, Sept. 2004, Nottingham, United Kingdom.

[8] Blaisot, J-B. and Abuzahra, F. and Sou, A. and Dumouchel, C., 2019,"Entropy-Based Cavitation and Primary Atomization Analysis with a 2D Transparent Injector", ILASS-Europe, Sept. 2019, Paris, France.

\section{Nomenclature}

$A_{a} \quad$ Primary atomization area

$A_{d} \quad$ Dispersed phase expansion area

$A_{j} \quad$ Liquid jet expansion area

$D_{g} \quad$ Coaxial injector outer gas diameter

$D_{l} \quad$ Coaxial injector inner liquid diameter

$H_{v} \quad$ Image height
$L_{a} \quad$ Primary atomization zone length

$L_{d} \quad$ Average dispersed phase zone length

$L_{j} \quad$ Average liquid jet length

$P_{s} \quad$ Probability of a given state

$P_{a c} \quad$ Acoustic pressure amplitude

$P_{\text {rad }} \quad$ Acoustic radiation pressure 